\title{
Inhalt, Vol. 3, 1980
}

Herausgeber Prof. Dr. S. Eckhardt, Staatliches Onkologisches Institut, Budapest

Prof. Dr. R. GROSS, Medizinische Universitätsklinik, Köln Prof. Dr. J. H. Holzner, Institut für pathologische Anatomie, Universität Wien Prof. Dr. K. Munk, Deutsches

Krebsforschungszentrum, Institut für Virusforschung, Heidelberg

Prof. Dr. G. P. Murphy, Roswell Park Memorial Institute, Buffalo (N.Y.) Prof. Dr. A. Stacher, Hanusch-Krankenhaus, Wien Prof. Dr. Dr. St. Tanneberger, Zentralinstitut für Krebsforschung, Akademie der Wissenschaften, Berlin/DDR

Prof. Dr. W. Wilmanns, Medizinische Klinik III, Klinikum Großhadern, Universität München Prof. Dr. Dr. H. Wrba, Institut für Krebsforschung, Universität Wien

Wissenschaftlicher H. W. Bauer, München; H. Denck, Wien; V. Diehl, Hannover; F. Douwes, Beirat Göttingen; P. Drings, Heidelberg; H. Ehrhart, München; J. Fischer, Mainz; W. M.

Gallmeier, Nürnberg; H. Gerhartz, Berlin; E. H. Graul, Marburg/Lahn; E. Haupt-mann, Zagreb; H. Heimpel, Ulm; K. P. Hellriegel, Köln; Ch. Herfarth, Ulm; R. Hünig, Basel; W. Hunstein, Heidelberg; H. O. Klein, Köln; H. Löffler, Lahn Gießen; U. Mohr, Hannover; F.-L. Müller, Bonn; G. Nagel, Göttingen; A. Pfleiderer, Freiburg; Mildred Scheel, Bonn; E. Scherer, Essen; S. Seeber, Essen; H. J. Senn, St. Gallen; W. Vahlensieck, Bonn; H. D. Waller, Tubingen; M.

Wannenmacher, Freiburg; K. Wilms, Tubingen; K. zum Winkel, Heidelberg

(C) Copyright 1980 by S. Karger Verlag für Medizin und Naturwissenschaften GmbH, Postfach 2,

D-8034 Germering/München

S. Karger · Basel · München · Paris · London · New York · Sydney

Inhalt

Seeber, S.; Niederle, N.; R. B. Schilcher und

C. G. Schmidt, Essen

Adriamycin, Cyclophosphamid und Vincristin (»ACO«)

beim kleinzelligen Bronchialkarzinom 5

Firusian, N., Essen

32P-Therapie des malignen Perikardergusses

Oeser, H. und W. Bohndorf

Das Larynkarzinom: Daten zur Prognose 18

Vutuc, Ch. und B. Gredler, Wien

Soziale Schicht und Bronchuskarzinom

Beck, A. und F. Nikorovicz, Wien

Das Sexualleben nach Radikaloperationen des Zervix-

karzinoms 26

Wöllgens, P.; Kuhne-Velte, H. J. und Franke-Lompa, C,

Darmstadt

Serum-Eisen und Serum-Kupfer-Relation bei der Früh-

erkennung von Metastasen des Mammakarzinoms .... 32

Riedler, L., Innsbruck 
Transanale lokale Tumorektomie

Onkologie-Mitteilungen 41

Bombik, B. M.; Oertel, J.; Schneider, D.; Gerhartz, H., Berlin

Chemotherapie des metastasierten Kolonkarzinoms mit

Nitroseharnstoffen 49

Haas, R. J.; Helmig, M.; Prentl, E., München

Effektivität intramuskulär verabreichten Cytosin-Ara-

binosids bei der ambulanten Behandlung von Kindern

mit akuter Leukämie in Remission 53

Havers, W.; Stollmann, B.; Bachmann, H. J.;

Bamberg, M.; Stambolis, C, Essen

Bestrahlungsbedingte Nierenerkrankung nach Behand

lung von Malignomen im Kindesalter $\quad$. . . 58

Sauer, Hansjörg; Schalhorn, Andreas, München

Rationale Grundlagen und Praxis des Citrovorumfaktor-

(Leucovorin ${ }^{\circledR}$ )-Schutzes nach hochdosierter Metho-

threxat ${ }^{\circledR}$-Therapie 64

Schmitt, G.; Scherer, E., Essen

Neutronentherapie am Radiologischen Zentrum des

Westdeutschen Tumorzentrums des Universitätsklini-

kums Essen 72

Sellschopp-Rüppell, Almuth, Heidelberg

Psychologische Betreuung von onkologischen Patienten . 74

Siemers, E.; Seeber, S.; Höffken, K.; Schmidt, C. G., Essen

Ergebnisse der zytostatischen Therapie mit Cyclophos

phamid, Vincristin, Adriamycin und DTIC (CYVADIC)

bei lokalisierten und metastasierten Osteosarkomen -

Eine retrospektive Analyse 78

Hinweise für Autoren $\quad 52$

Letzte Meldungen 62

Buchbesprechungen $\quad 85$

Onkologie-Mitteilungen

Rhomberg, W., Hannover

Probleme der kumulativen Toxizität von Radiotherapie

und zytostatischer Chemotherapie beim Bronchial

karzinom 97

Drings, P., Heidelberg

Durchführung und Problematik des Staging bei Bron-

chialkarzinomen 104

Vogt-Moykopf, I., Lüllig, H. und Toomes, H., Heidelberg

Operativer Stand und Möglichkeiten beim Bronchial

karzinom 112

Havemann, K., Marburg/Lahn

Immuntherapie beim Bronchialkarzinom 120

Müller, K.-M., Münster 
Problematik der histologischen Klassifikation des Bronchialkarzinoms $\quad 127$

Gropp, C. und Havemann, K., Marburg/Lahn

Bedeutung von Tumormarkern in der Diagnostik und

Behandlung des Bronchialkarzinoms . 133

Buchbesprechungen 139

Onkologie-Mitteilungen 140

Kraus-Sandmair, B. und Dinges, H. P., Wien

Untersuchungen zur Problematik einer jatrogenen In-

duktion von Mehrfachmalignomen anhand eines größe-

ren Autopsiegutes 157

Husemann, B.; Neubauer, M. G. und Duhme, C,

Erlangen

Sitzende Tätigkeit und Rektum-Sigma-Karzinom .... 168

Ries, P. und Reichert, P., Hannover

Ein Beitrag zur allgemeinen und speziellen Pathologie

der benignen odontogenen Tumoren 172

Bauer, H.-W., München

Das schwangerschaftsassoziierte ou-Glykoprotein

(012 PAG). - Ein möglicher Marker zur Tumorverlaufs-

kontrolle? 183

Kokron, O.; Olbert, F. und Schlegl, A., Wien

Intraarterielle Infusionsbehandlung von Lungentumoren 187

Schalhorn, A.; Wilmanns, W. und Stupp-Poutot, G.,

München

Methotrexat-Nachweis im menschlichen Serum - Ver-

gleich zwischen einer enzymatischen und einer enzym-

immunologischen Methode 193

Buchbesprechungen 197

Onkologie-Mitteilungen 198

Buchbesprechungen 212

Vorwort 213

Adolphs, H.-D.; Thiele, J. und Vahlensieck, W.,

Hannover/Bonn

Epidemiologie und Pathogenese des Harnblasenkarzi-

noms 214

Rübben, H.; Dahm, H. H. und Lutzeyer, W., Aachen

Klinische Diagnostik beim Blasenkarzinom 225

De Voogt, H.J., Amsterdam

Bedeutung der Urinzytologie als Screening-Untersuchung

für die Diagnose eines Harnblasenkarzinoms 233

Oehr, P. und Adolphs, H.-D., Bonn

Bedeutung von biologischen Markersubstanzen für die

Diagnose des Harnblasenkarzinoms 238 
Thüroff, J. W. und Jacobi, G.H., Mainz

Therapie, Nachbehandlung und Prophylaxe des Harn

blasenkarzinoms 248

Onkology-Mitteilungen 264

Maier, U.; Kuber, W. und Krepler, R., Wien

Wertigkeit der Exfoliativzytologie des Harns bei der

Diagnose von Blasentumoren 276

Tribukait, B. und Gustafson, H., Stockholm

Impulscytophotometrische DNS-Untersuchungen bei

Blasenkarzinomen 278

Possinger, K.; Hartenstein, R. und Ehrhart, H., München In vitro-Resistenztestung von Tumoren

gegenüber Zyto-statika - 1. Tierexperimentelle Voruntersuchungen -2. Untersuchungen an

menschlichen Malignomen .... 291

Francesconi, M.; Mörz, R.; Rainer, H. und Moser, K.,

Wien

Phase-2-Studie mit cis-Dichlorodiaminoplatinum (II) bei

weit fortgeschrittenen soliden Tumoren 301

Kamprad, F.; Pfeiffer, J. und Oelssner, W., Leipzig

Zur Fernmetastasierung intensiv behandelter Harn-

blasenkarzinome $\quad 307$

v. Kleist, S.; Wagener, Ch. und Breuer, H., Freiburg

2. Internationales Expertentreffen der Deutschen Stiftung für Krebsforschung »Current Trends in Cancer

Research« 310

Köstering, H. und Nagel, G. A., Göttingen

Prophylaxe und Therapie von Zytostatika-Hautnekrosen 317

Buchbesprechungen 321

Onkologie-Mitteilungen 